\title{
Editorial
}

\section{Improving quality of drug use through hospital directorates}

Drugs are important to patients and to the NHS for three reasons. Firstly, drugs can increase the quality and duration of life; often they are the only way of doing so. Secondly, their use is associated with a risk that they may have the opposite effect. Finally, because society values them, they have a high volume of use; and can have a high cost, so they are an important expenditure for the NHS. Indeed, after staff costs, drug costs account for the highest expenditure in the NHS, and the government considers that controlling their use is a priority. The quality of drug use (prescribing, supply, administration, and adherence to treatment) must therefore be a priority.

\section{Existing framework for quality assurance}

The relationship between a pharmacy and a drug and therapeutics committee forms the backbone of the quality assurance mechanisms for drug use in hospitals. The committee generally issues policies and protocols; the pharmacy monitors drug use by retrospective analysis of its data on purchasing and issue of drugs and by daily observation of prescribing and drug use in wards. Because the pharmacy purchases drugs, holds the drugs budget, and has a computerised issuing system, many of its measures to improve the quality of drug use were originally driven by the need to reduce expenditure. The ability of pharmacies to target areas of high expenditure led to the development of drug use reviews. In Britain this has usually involved a drug, or therapeutically equivalent group of drugs, being studied in depth to ensure that their use was appropriate and that cheaper alternatives were not available. Although the reduction of expenditure was often the initial stimulus, these reviews also concentrated on safety, ease of use for nurses or patients, or patient acceptability.

Although results of drug use review studies, "top 50" drugs, and formulary issues are discussed at drug and therapeutics committees, the second part of the pharmacists' activities - namely, monitoring prescribing and drug use in the wards - has never been directed into the pharmacy-drug and therapeutics committee quality assurance mechanism systematically. There is a significant activity which usually goes no further than a discussion between a ward pharmacist and junior doctor or nurse. For example, North West Thames Regional Health Authority conduct a survey of ward pharmacists' activity annually; in 1992 the results showed that 2530 prescriptions were changed in a week on the advice of pharmacists. The drug and therapeutics committee is usually unaware of the extent (or appropriateness) of this advice, as is the consultant whose patients' prescriptions have been changed. What is more, training needs and trends in behaviour are not observed because this information is not aggregated. Now is the time for change. Pharmacists are developing ways of systematically monitoring ward pharmacists' activities. ${ }^{1-3}$ Most of these systems are computerised and produce a monthly summary of the pharmacists' performance, which is used for management and training. The systems can also categorise interventions by consultant or directorate to identify trends in drug use.

\section{Time for change}

It is time for a new model for quality assurance of drug use in hospitals, one that brings together pharmacists, prescribers, nurses, and others to examine all aspects of drug use. The clinical directorate is the logical unit around which to base such a model. The structure is already in place, as the clinical director usually meets monthly with a pharmacist who advises on the figures for drug expenditure and usage. This meeting could become the basis of a new interprofessional group for quality assurance of drugs (QUAD). In addition to matters arising from the monthly figures for expenditure and usage, the group could receive a summary of ward pharmacists' interventions and their outcome. This overview of drug use would drive policies and practices within the directorate. In addition, there could be a 12 month rolling cycle of clinical audit issues related to the prescribing, supply, and administration of drugs and discharge arrangements. The discussions could be held monthly for some topics - for example, drug expenditure and prescribing - and quarterly for others such as supply and manner of usage. Supply could be measured by markers such as missed doses, errors of administration by nurses, and delayed discharge of patients because they were waiting for medicines. Appropriate use of medicines could include consideration of the intravenous route (diluents, speed of bolus injections, line failures, co-administration of drugs, and existence of an alternative route); the use of nebulisers, medical gases, dressings, and urine testing strips; and instances of dysphagic patients made to struggle with tablets when a liquid form of the drug was available.

The quality group would take on many of the tasks currently performed by the drug and therapeutics committee but would not supplant it. The figure shows the structure envisaged and how the groups would work in parallel with the existing pharmacy - drug and therapeutics committee axis. The role of the drug and therapeutics committee, however, would change to one of overview, coordination of the quality groups, and arbitration between them on conflicts of policy.

Why should change be beneficial?

Why should this structure ensure a better quality of drug use than the current system? The solution to problems

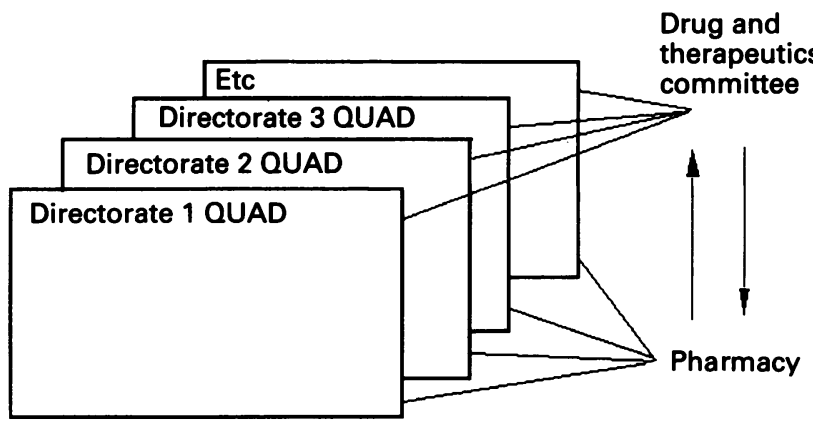

Directorate based groups for quality assurance of drugs (QUADs) and their relationships with drug and therapeutics committee and pharmacy department 
of quality often lies in one or more of four areas: education, the systems of work, management, and the working environment or equipment. Pharmacy has been successful because it has used all four areas, but it has limited power. Medicine tends to use education, a technique that is not always successful in creating change. ${ }^{4}$ The hierarchical and expert power of clinical directors, together with their ability to use virement and manage a coordinated multidisciplinary team, makes them powerful agents of change. For the first time, all information relevant to drug use would be focused in one group; what is more, that group is small enough to have ownership and powerful enough to create change. The formation of directorate based quality groups could have a considerable effect on the quality of drug use to the benefit of patients, purchasers, and provider units alike.

Professor of the Practice of Pharmacy,

NICK BARBER

School of Pharmacy,

University of London, London WC1N $1 A X$

1 Cousins D, Hatoum $\mathrm{H}$. The development of a computerised quality assurance system for clinical pharmacy. Int f Pharm Prac 1991; 1:86-9.

2 Kennedy $\mathrm{K}$, Wind $\mathrm{K}$. The development of a monitoring package for pharmacy interventions. Hosp Pharm Prac 1992;2:93:101.

3 Batty B, Barber ND. Ward pharmacy: a foundation for prescribing audit? Quality in Health Care 1992;1:5-9.

4 Davis DA, Thomson AM, Oxman AD, Haynes RB. Evidence for the effectiveness of CME. A review of 50 randomized trials. $\mathscr{f} A M A 1992$; 268:111-7.

过 